\title{
Correlations between P2RX1 Expression and Gastrointestinal Cancers Prognosis and Immune Infiltration Based on Bioinformatics Analysis
}

\author{
Ting Li', Jiankai Feng2, Pihong Yan², Hongwei Tan², Lili Wang2, Qiang Li', Yunliang Yu2* \\ ${ }^{1}$ Department of Pathology, Yantai-Mountain Hospital of Yantai, Yantai 264000, Shandong Province, China \\ ${ }^{2}$ Department of Medical Laboratory, Yantai Affiliated Hospital of Binzhou Medical University, Yantai 264000, Shandong \\ Province, China
}

*Corresponding author: Yunliang Yu, 6667943.good@163.com

\begin{abstract}
This study was conducted to explore the correlations between the expression, methylation, and various clinicopathological factors of purinergic P2X1 receptor (P2RX1) and the prognosis of patients with gastrointestinal tumors. The Cancer Genome Atlas (TCGA) and the Genotype-Tissue Expression (GTEx) databases were used to analyze the expression of P2RX1 in different types of gastrointestinal cancers. Kaplan-Meier analysis and univariate Cox regression analysis were used to analyze the correlations between P2RX1 expression and the prognosis of various gastrointestinal tumors. Correlations between P2RX1 expression and N6 methyladenine (m6A)-related genes as well as immune checkpoint genes were analyzed by R packages ( $R$ version: 4.0.3) based on TCGA database. The association between P2RX1 methylation level and the prognosis of patients with gastrointestinal cancers was analyzed using the MethSurv database. In order to explore the biological functions of P2RX1 in hepatocellular carcinoma, the Kyoto Encyclopedia of Genes and Genomes (KEGG) and Gene Ontology (GO) enrichment analysis were carried out using R software. In order to evaluate the correlations between P2RX1 and tumor immune infiltration, Spearman correlation test was performed. The correlations between P2RX1 expression and immune score as well as immune checkpoint genes were analyzed based on TCGA and Tumor Immune Estimation Resource (TIMER) databases. The expression of P2RX1 was found to be significantly downregulated in gastrointestinal tumors except in cholangiocarcinoma $(P<0.05)$. High expression of P2RX1 tended to present better prognosis in hepatocellular carcinoma $(P<0.05)$. It was noted that $\operatorname{cg} 06475633$ of $\mathrm{P} 2 \mathrm{RX} 1$ presented a higher methylation level compared with other $\mathrm{CpG}$ sites in hepatocellular carcinoma. Overall, six CpGs of P2RX1 were associated with significant prognosis in patients with hepatocellular carcinoma $(P<0.05)$. Among all the $20 \mathrm{~m} 6 \mathrm{~A}$-related genes, Wilms' tumor 1 -associating protein (WTAP) was the most strongly correlated with P2RX1 in hepatocellular carcinoma. Gene enrichment analysis showed that P2RX1 is widely involved in the proliferation, activation, organization, and differentiation of various immune cells. After investigating the TIMER database, P2RX1 was found to be tightly correlated with immune infiltrating cells in gastrointestinal tumors, especially with dendritic cells. Moreover, P2RX1 was found to be strongly positively associated with programmed cell death 1 (PD1), programmed death-ligand 1 (PD-L1), and cytotoxic T-lymphocyte-associated protein 4 (CTLA4) in hepatocellular carcinoma $(P<0.05)$. In conclusion, the dual role of P2RX1 in cancers and its involvement in the recruitment as well as regulation of tumor infiltrating cells in gastrointestinal cancers may be appreciated through this study.
\end{abstract}

Keywords: P2RX1; Gastrointestinal cancers; Prognosis; Methylation; Tumor immune infiltration

Publication date: November 2021; Online publication: November 30, 2021

\section{Introduction}

As the majority of cancer-related deaths in the world, gastrointestinal cancers account for one of the most 
common malignancies with increasing incidence and mortality ${ }^{[1,2]}$. For advanced gastrointestinal cancers, the primary treatment is through surgery combined with chemoradiotherapy, but this treatment often leads to unsatisfactory outcomes ${ }^{[3]}$. Fortunately, as immune-related mechanisms play critical roles in various gastrointestinal cancers, immunotherapy has revolutionized cancer treatment ${ }^{[4]}$. Gastrointestinal cancers are typically considered to be highly immunogenic, and immunotherapy is currently reckoned as one of the first-line treatment for patients with advanced cancer ${ }^{[5]}$. Nevertheless, there are numerous patients with advanced gastrointestinal cancer who are unresponsive to immunotherapeutic agents, and the side effects upon receiving this treatment still exist ${ }^{[6]}$. The treatment effectiveness of immunotherapy is unsatisfactory, owing to tumor heterogeneity and specificity ${ }^{[7]}$. Therefore, in order to improve the prognosis of gastrointestinal cancers, it is essential to explore better therapeutic modalities and mechanisms.

ATP receptors are extensively found on the surface of immune cells that infiltrate into the tumor microenvironment (TME). Extracellular ATP can bind to its receptors and directly affect cancer progression ${ }^{[8]}$. There are seven members in the ATP gated ion channel receptor family, of which P2RX1 is closely related to various biological processes, such as energy metabolism, immune response, and tumor metastasis

[9]. Studies have shown that P2RX1 acting on acidic nuclear phosphoprotein 32 family member A (ANP32A) can affect the enrichment of acetylation regulatory molecule $\mathrm{H} 3$ and promote the occurrence of leukemia ${ }^{[10]}$. High levels of P2RX1 expression tend to present better prognosis for patients with lung adenocarcinoma ${ }^{[11]}$. Moreover, P2RX1 was found to be tightly correlated with the differentiation and development of B cells and mast cells in TME. In liver metastatic pancreatic ductal adenocarcinoma, P2RX1 was found to be associated with ATP-mediated apoptosis signaling pathway, and the downregulation of P2RX1 promoted tumor cells escape from anti-tumor immunity by regulating and recruiting neutrophils ${ }^{[12]}$. In P2RX1negative neutrophils, the transcription factor NF-E2 p45-related factor 2 (Nrf2)-supported mitochondrial metabolism was found to be tightly correlated with the reshaping of neutrophils' immunosuppressive phenotypes ${ }^{[13]}$. In inflamed colon tissues, the expression of P2RX1 was found to be upregulated; however, a reduction in P2RX1 expression could suppress inflammation responses in dextran sulfate sodium (DSS)induced colitis in mice ${ }^{[14]}$.

Currently, report on whether P2RX1 is involved in the progression of gastrointestinal tumors remains unclear, and its abnormal expression in tumors has not been systematically studied. In this study, different bioinformatics tools were employed to comprehensively analyze the P2RX1 expression level in patients with cholangiocarcinoma (CHOL), colon adenocarcinoma (COAD), esophageal carcinoma (ESCA), liver hepatocellular carcinoma (LIHC), pancreatic adenocarcinoma (PAAD), rectum adenocarcinoma (READ), and stomach adenocarcinoma (STAD). The expression and prognostic value of P2RX1 in gastrointestinal tumors were further explored. The results indicated that P2RX1 was significantly deregulated in almost all the types of gastrointestinal cancers. P2RX1 expression level was found to be significantly correlated with survival rates, tumor infiltrating lymphocytes (TILs), immune checkpoint genes, and immune/stromal scores of patients with LIHC. In addition, P2RX1 may influence methylation in LIHC. From the results, P2RX1 may be selected as a potential target for immunotherapy, especially in low-response tumors.

\section{Methods}

\subsection{P2RX1 expression analysis}

The transcriptome data generated from the gastrointestinal tumors dataset and clinical data of patients were downloaded from The Cancer Genome Atlas database (TCGA), https://portal.gdc.cancer.gov/, and the Genotype-Tissue Expression project database (GTEx), http://genome.ucsc.edu/gtex.html. The quantification data of P2RX1 expression patterns for transcriptome profiling comprised of $36 \mathrm{CHOL}$ patients, 457 COAD patients, 184 ESCA patients, 371 LIHC patients, 178 PAAD patients, 166 READ 
patients, and 415 STAD patients.

\subsection{Cox regression analysis and survival analysis}

The clinicopathological data of gastrointestinal tumors were downloaded from TCGA and GTEx databases. Based on univariate Cox regression analysis, the clinical data were analyzed by $\mathrm{R}$ packages, including "survival" and "forestplot." The Kaplan-Meier algorithm was also employed to establish their cumulative survival curves. In order to identify the significance of difference, the log-rank method was performed. Correlations between the expression level of P2RX1 and the overall survival (OS) rates, disease-specific survival rates (DSS), disease-free interval (DFI) rates, and progression-free interval (PFI) rates of patients with gastrointestinal tumors were analyzed. Correlations between the expression of P2RX1 and different clinicopathological factors such as age, gender, race, TNM stage, and grade was analyzed by R packages, "ggalluval" and "ggplot2."

\subsection{DNA methylation analysis}

The methylation data of P2RX1 in gastrointestinal tumors were downloaded from TCGA database. Correlations between P2RX1 and four DNA methyltransferases (DNMT1, DNMT2, DNMT3A, and DNMT3B) were also analyzed based on TCGA database. The online public MethSurv database, from https://biit.cs.ut.ee/methsurv/, was used to cluster the methylation levels of different CpG sites of P2RX1. The associations between the methylation patterns of P2RX1 and different clinicopathological characteristics (nationality, race, BMI index, age, and survival) of LIHC patients were calculated using the MethSurv database. A forest map of the correlations between each single CpG site of P2RX1 and the overall survival rates of LIHC patients was drawn by using R package, "forestplot." The R packages - "ggstatsplot" and "pheatmap" were integrated to analyze the correlations between P2RX1 and m6A-related genes.

\subsection{Gene set enrichment analysis}

The P2RX1 expression and transcriptome data in LIHC with clinical information were obtained from TCGA database. The mRNA expression datasets of P2RX1 were separated into P2RX1 ${ }^{\text {high }}$ and $\mathrm{P} 2 \mathrm{RX} 1^{\text {low }}$ expression groups according to the quartile (25\%) of P2RX1 mRNA expression level in LIHC. Differential expression genes (DEGs) between P2RX1 ${ }^{\text {high }}$ and P2RX1 ${ }^{\text {Low }}$ groups were analyzed by R package, "Limma". For DEGs screening, " $P<0.05$ " and " $\log _{2} \mathrm{FC}>1$ " methods were identified in order to set thresholds. The upregulated and downregulated genes of P2RX1 expression in LIHC were screened to perform gene set enrichment analysis. For detecting differential expression cluster, the "DEseq2" package in R was explored. The enrichment analysis of the above DEGs was performed by using "ClusterProfiler," "ggplot2," "circle," "enrich," and other R packages. Gene Ontology (GO) function analysis and Kyoto Encyclopedia of Genes and Genomes (KEGG) for differentially expressed genes pathway were analyzed.

\subsection{Immune infiltration analysis}

Correlations between P2RX1 expression and different kinds of TILs (B cells, $\mathrm{CD}_{4}{ }^{+} \mathrm{T}$ cells, $\mathrm{CD}_{8}{ }^{+} \mathrm{T}$ cells, macrophages, neutrophils, and dendritic cells) in the TME of gastrointestinal tumors were analyzed using $\mathrm{R}$ software based on TCGA and GTEx databases. For further identification, correlations between the expression of P2RX1 and immune cells were also verified by the Tumor Immune Estimation Resource (TIMER) 2.0 database. The estimation of stromal and immune cells in malignant tumor tissues using expression data (ESTIMATE) computational algorithm was employed to estimate the proportion of 
TILs and the degree of infiltration of immune and stromal components based on TCGA database.

\subsection{Statistical analysis}

In order to identify the expression pattern of P2RX1 between tumor tissues and adjacent normal tissues, Student's $t$-test was employed. Associations between P2RX1 expression and different clinicopathological factors of patients with LIHC were calculated by employing the chi-squared test. In order to identify the associations of P2RX1 with 20 m6A-related genes, DNA methyltransferases (DNMTs), immune infiltrating scores, immune checkpoint genes, and immune cells infiltration in different cancer types, Spearman correlation test was performed. R packages were employed to perform statistical analysis, including "ggplot2," "forestplot," "ggstatsplot," "ggalluval," "maftools," "enrichplot," "ClusterProfiler," and "pheatmap" projects. A $P$-value lower than 0.05 was considered significant.

\section{Results}

\subsection{P2RX1 is downregulated in gastrointestinal tumors}
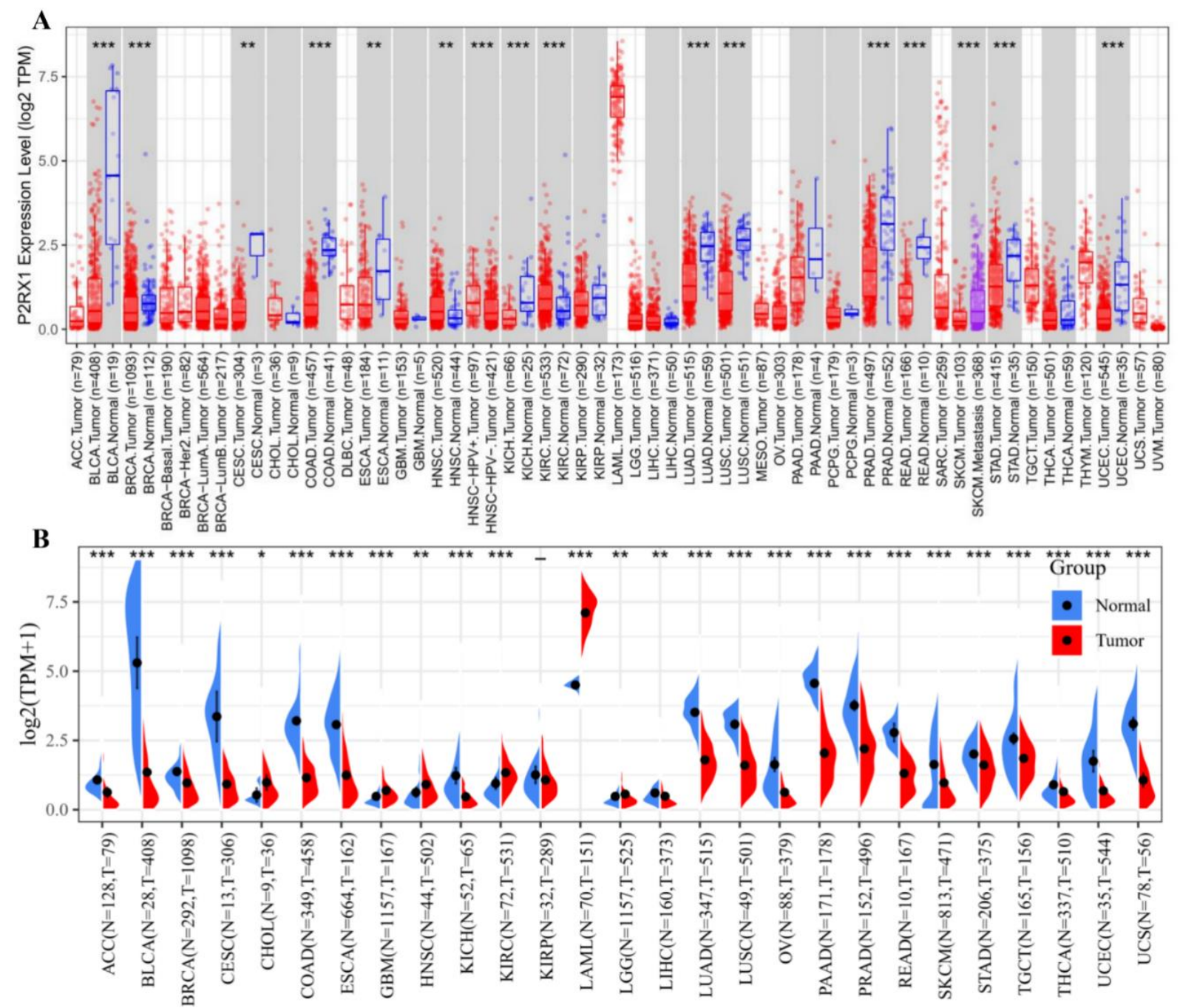

Figure 1. P2RX1 expression pattern in different types of cancers. (A) P2RX1 expression level in different tumors from TIMER database. (B) Comparing different tumor tissues with normal tissues, P2RX1 expression level was analyzed based on TCGA and GTEx databases. Note: $* P<0.05 ; * * P<0.01 ; * * P<0.001$. 
In order to identify the P2RX1 expression pattern in different gastrointestinal tumors, the TIMER database was first investigated based on TCGA data. The results showed that P2RX1 was downregulated in COAD, ESCA, LIHC, PAAD, READ, and STAD (Figure 1A). However, the expression of P2RX1 in CHOL tumor tissues and adjacent normal tissues had no significant difference. Subsequently, TCGA and GTEx databases were integrated by $\mathrm{R}$ software to further analyze the expression pattern of P2RX1 in gastrointestinal tumors. The findings also showed that the expression of P2RX1 was significantly downregulated in the aforementioned six gastrointestinal tumors - COAD, ESCA, LIHC, PAAD, READ, and STAD (Figure 1B).

\subsection{Low expression of P2RX1 is associated with the prognosis of patients with LIHC, PAAD, and READ}

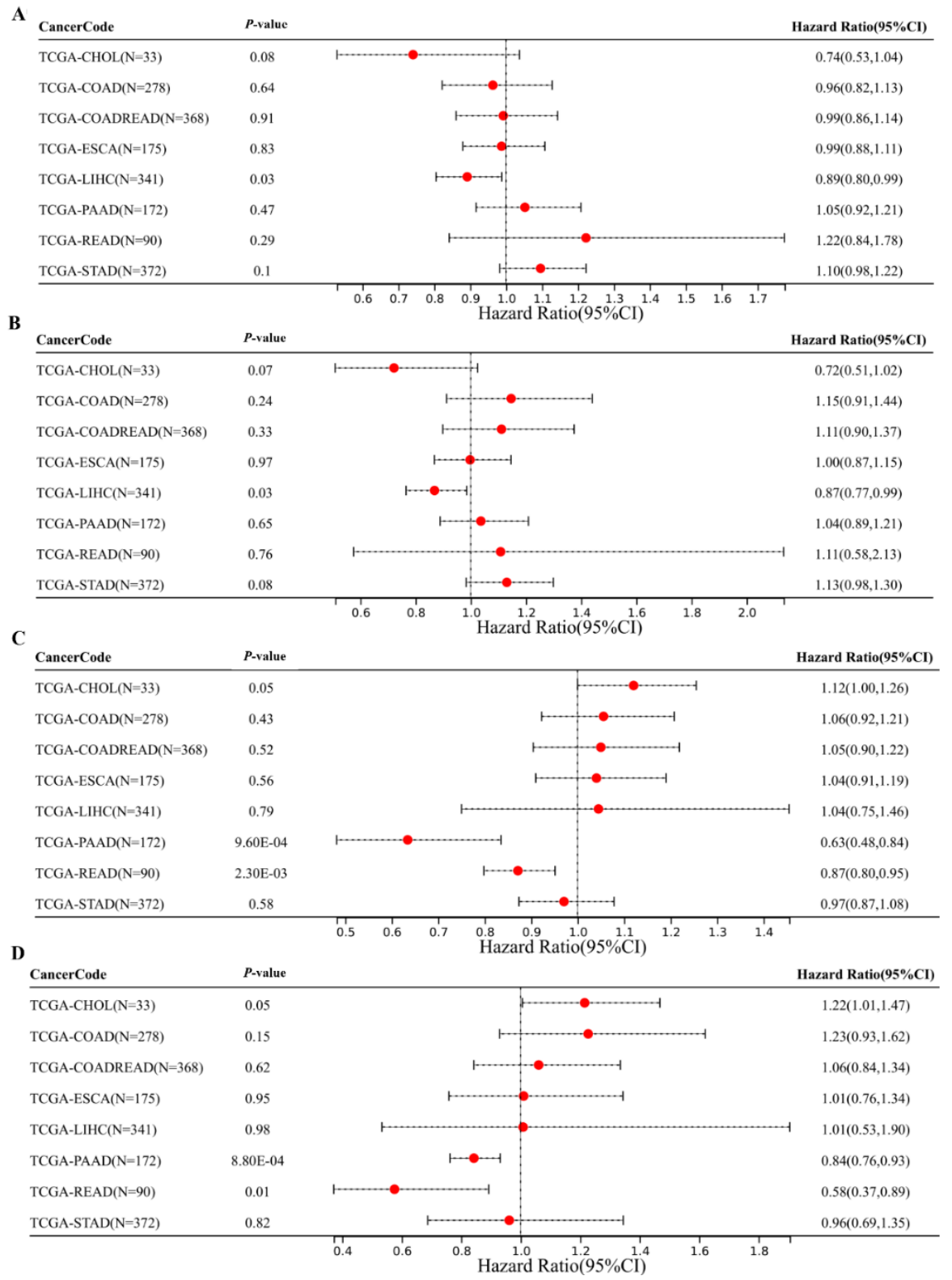

Figure 2. Correlations between P2RX1 expression and the prognosis of patients with eight different types of gastrointestinal cancers. (A) The effect of P2RX1 on the overall survival rate in eight types of gastrointestinal cancers. (B) The effect of P2RX1 on the disease-specific survival rate in eight types of gastrointestinal cancers. (C) The effect of P2RX1 on the disease-free interval rate in eight types of gastrointestinal cancers. (D) The effect of P2RX1 on the progression-free interval rate in eight types of gastrointestinal cancers. 
The above results suggest that P2RX1 may act as a tumor inhibitor in gastrointestinal cancers and the downregulation of P2RX1 may worsen the prognosis of these cancers. In order to validify the speculation, Cox univariate regression analysis was employed to analyze the clinical data of patients with gastrointestinal tumors based on TCGA database. The forest plots showed that the hazard ratios for P2RX1 were significant for LIHC (Figure 2A). P2RX1 also had correlations with the DSS rate (Figure 2B) and DFI rate (Figure 2C) in LIHC. In patients with PAAD and READ, the expression of P2RX1 was also found to be significantly correlated with DFI (Figure 2C) and PFI (Figure 2D).

\subsection{Correlations between P2RX1 and various clinicopathological factors of LIHC}
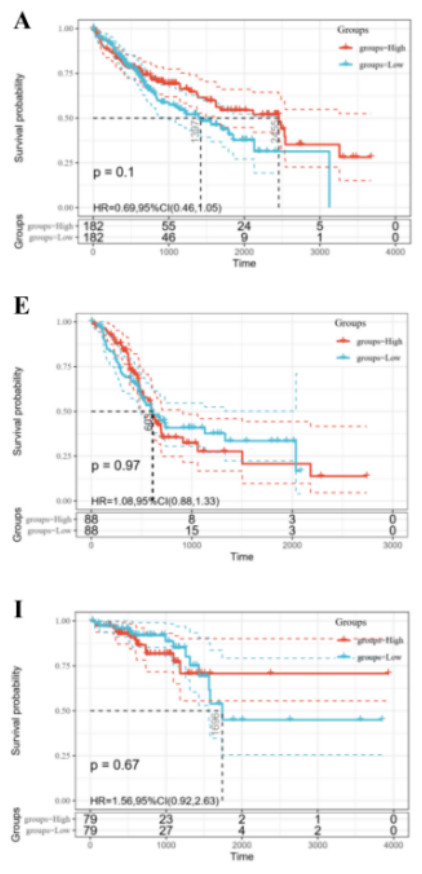

M

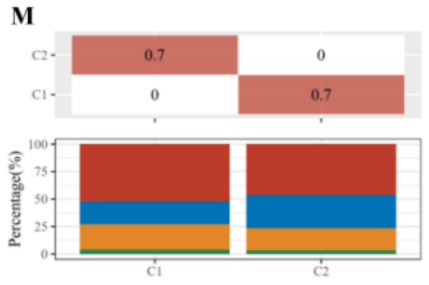

$\mathbf{Q}$
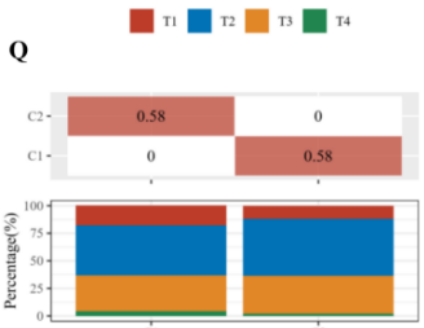

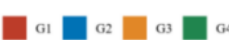
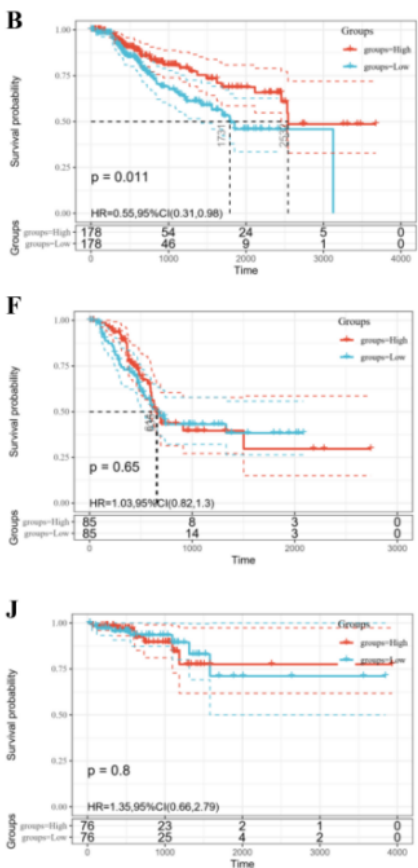

$\mathbf{N}$

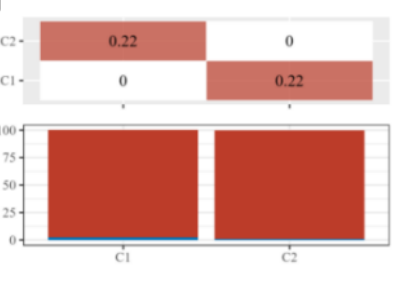

$\mathbf{R}$

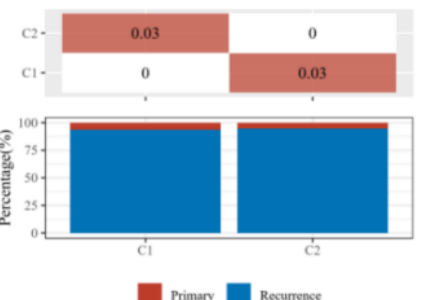

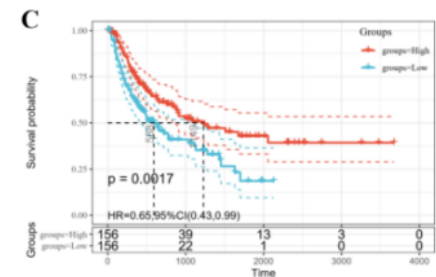
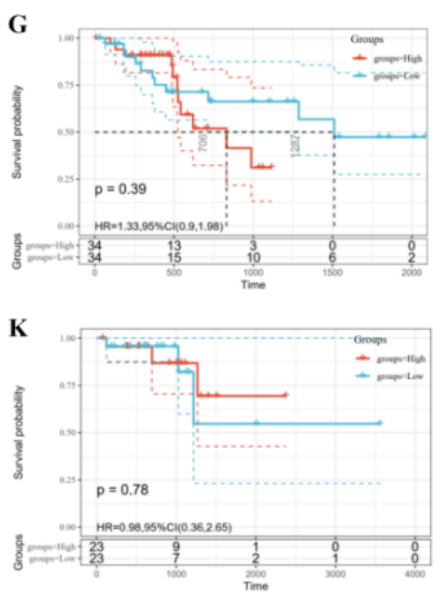

o

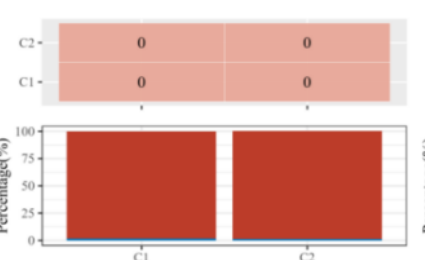

S

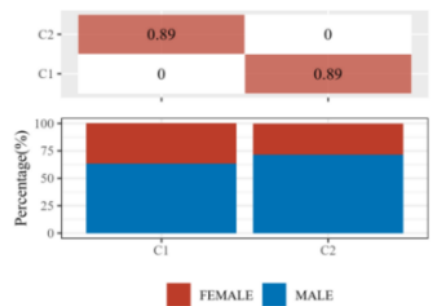

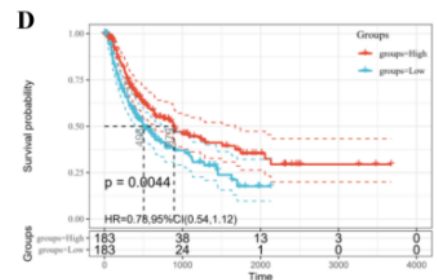

H
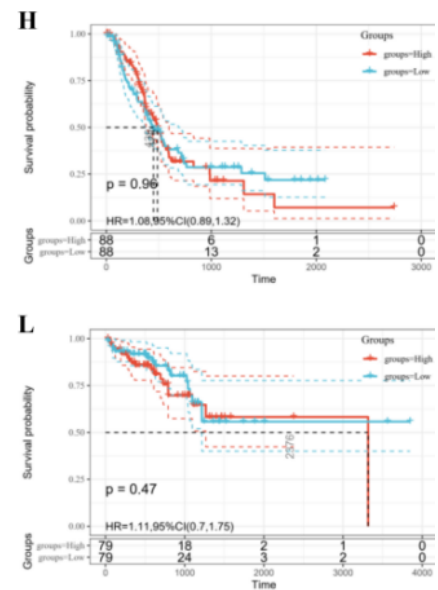

P

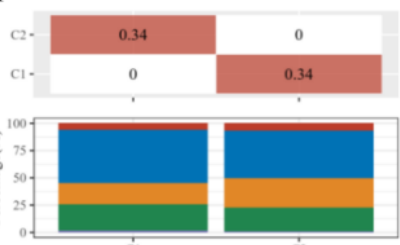

T

$\square \square \cdot \square \mathrm{nm} \mathbf{m}$

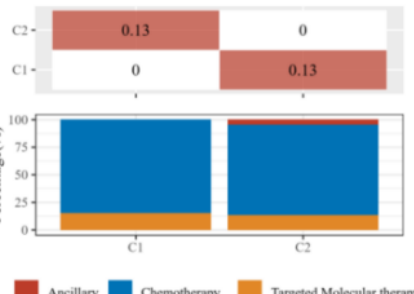

Figure 3. Correlations between $P 2 R X 1$ expression and the survival rates of patients with LIHC, PAAD, and READ. (A-D) The effect of P2RX1 on OS, DSS, DFI, and PFI in LIHC. (E-H) The effect of P2RX1 on OS, DSS, DFI, and PFI in PAAD. (I-L) The effect of P2RX1 on OS, DSS, DFI, and PFI in READ. (M-T) Correlations between P2RX1 and T stage, N stage, M stage, clinical stage, grade, tumor types, gender, and treatment in LIHC, respectively. Note: C1, P2RX1 low expression group; C2, P2RX1 high expression group. 
The Kaplan-Meier analysis showed that no significant association was discovered between P2RX1 and the OS of patients with LIHC (Figure 3A). On the contrary, P2RX1 had significant associations with the DSS (Figure 3B), DFI (Figure 3C), and PFI (Figure 3D) of patients with LIHC. There were no significant associations between P2RX1 and the OS (Figure 3E), DSS (Figure 3F), DFI (Figure 3G), and PFI (Figure 3H) of patients with PAAD. Similarly, between P2RX1 and the OS (Figure 3I), DSS (Figure 3J), DFI (Figure 3K), and PFI (Figure 3L) of patients with PAAD and READ, no significant associations were discovered. After exploring the correlations between P2RX1 and various clinicopathological factors of LIHC, it was found that there were no significant differences between P2RX1 and the TNM stage, grade, therapy, gender, and clinical stage of patients with LIHC (Figure 3M to Figure 3T).

\subsection{P2RX1 methylation is linked to the prognosis of patients with LIHC}

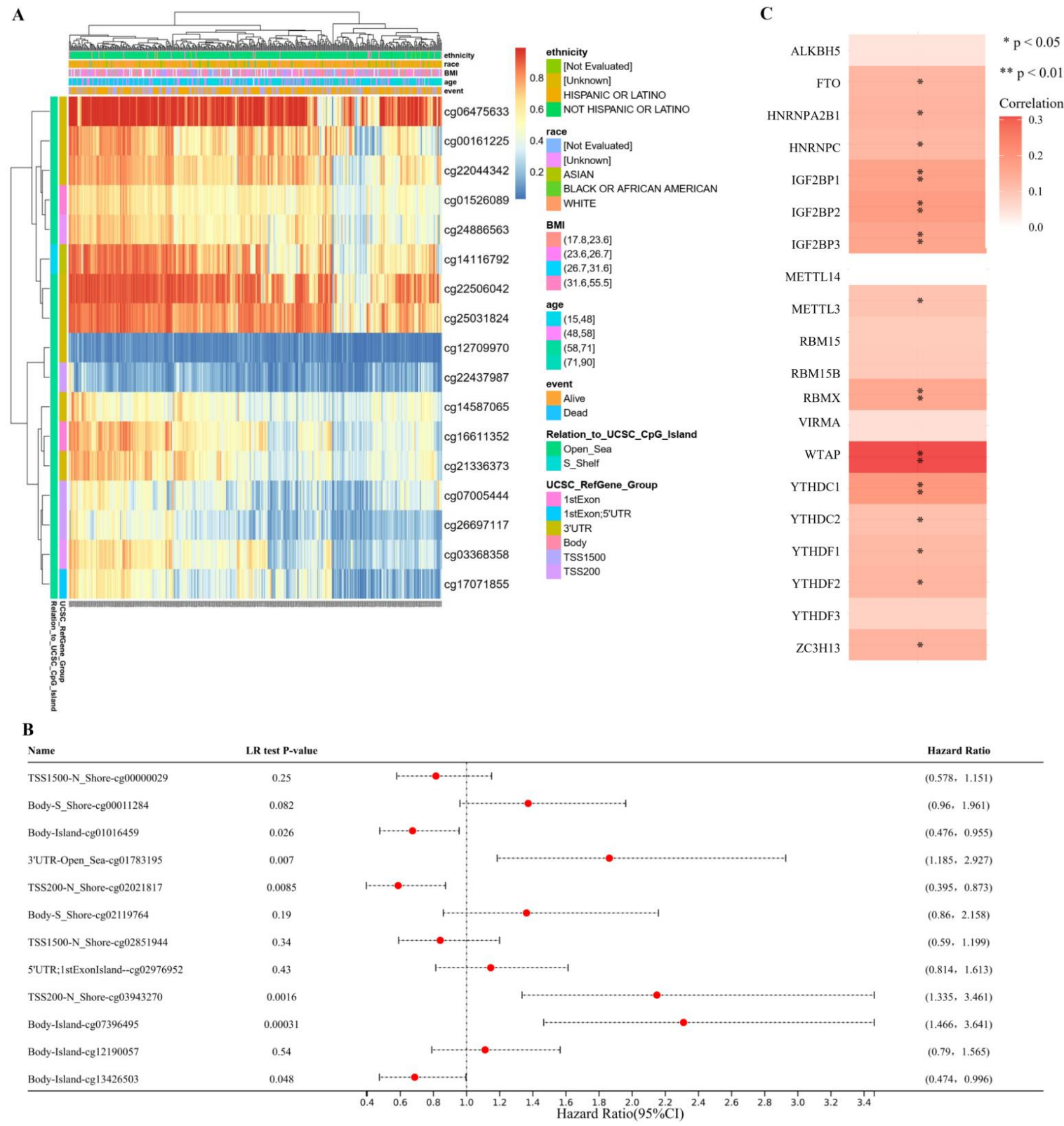

Figure 4. Correlations between P2RX1 and DNA methylation level as well as m6A-related genes in LIHC. (A) The DNA methylation expression of P2RX1 in LIHC. (B) The prognostic values of different P2RX1-CpGs in LIHC. (C) Correlations between P2RX1 and 20 m6A-reltaed genes in LIHC. Note: $* P<0.05 ; * * P<0.01$. 
The above results show that the downregulation of $\mathrm{P} 2 \mathrm{RX} 1$ is correlated with poor prognosis in patients with LIHC. The associations between P2RX1 expression and DNMTs were further explored based on TCGA database. P2RX1 was found to be positively correlated with DNMT1 $(r=0.19, P=0.00068)$, DNMT2 $(r=0.23, P=1.9 \mathrm{e}-5)$, and DNMT3A ( $r=0.14, P=0.017)$ in LIHC. To further investigate the clinical significance of P2RX1, the correlations between the DNA methylation level of P2RX1 and the prognostic values of each single were analyzed by MethSurv database. The methylation level of P2RX1 was found to be associated with different clinicopathological characteristics (nationality, race, BMI, age, survival, etc.) of LIHC patients. In this study, each single CpG of P2RX1 was clustered in the form of heat map. It showed that cg06475633 presented a higher methylation level compared with other $\mathrm{CpG}$ sites in LIHC (Figure 4A). Subsequently, the associations between the methylation level of each single CpG of P2RX1 and the prognosis of patients with LIHC were further analyzed based on TCGA database. Among all the 12 CpG sites, cg0101649, cg01783195, cg02021817, cg03943270, cg07396495, and cg13426503 were found to be correlated with the overall survival rates of patients with LIHC (Figure 4B). As the most prevalent modification of RNA methylation, the m6A modification is a dynamic and reversible process, and it regulates the expression of proto-oncogenes as well as tumor suppressor genes by affecting specific RNA molecules in tumors. In this research, the correlations between P2RX1 and twenty m6A-related genes in LIHC were further investigated. P2RX1 was found to be positively correlated with FTO, HNRNPA2B1, HNRNPC, IGF2BP1, IGF2BP2, IGF2BP3, METTL3, RBMX, WTAP, YTHDC1, YTHDC2, YTHDF1, YTHDF2, and ZC3H13 in LIHC (Figure 4C). Among all the 20 m6A RNA methylation genes, P2RX1 was most strongly correlated with WTAP in LIHC $(r=0.34, P=1.6 \mathrm{e}-11)$.

\subsection{P2RX1 is widely involved in immunomodulatory functions and mechanisms in LIHC}

Data from TCGA were separated into $\mathrm{P} 2 \mathrm{RX} 1^{\text {high }}$ and $\mathrm{P} 2 \mathrm{RX} 1^{\text {low }}$ expression group for further enrichment analysis, in order to have a deeper insight about the gene functions of P2RX1 in LIHC. According to the above screening conditions, the upregulated genes and downregulated genes were screened. As shown by the volcano plot and heatmap, differential gene expression analysis identified 1,100 gene reporters that were upregulated and 36 gene reporters that were downregulated at least 2-fold (Figure 5A and Figure 5B). The enrichment analysis of the DEGs showed that those genes were mostly involved in viral protein interaction with cytokine receptor pathway, viral myocarditis pathway, tuberculosis pathway (Figure 5C). The DEGs enriched in the regulation of mononuclear cell proliferation, $\mathrm{T}$ cell activation, regulation of lymphocyte proliferation, and the regulation of leukocyte proliferation (Figure 5D). 
A

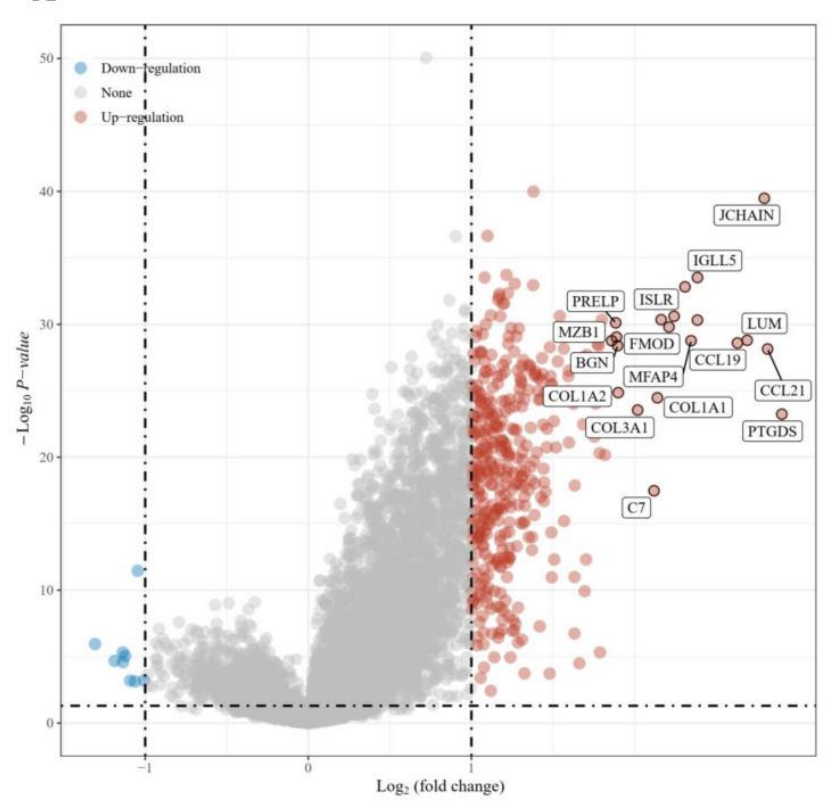

C

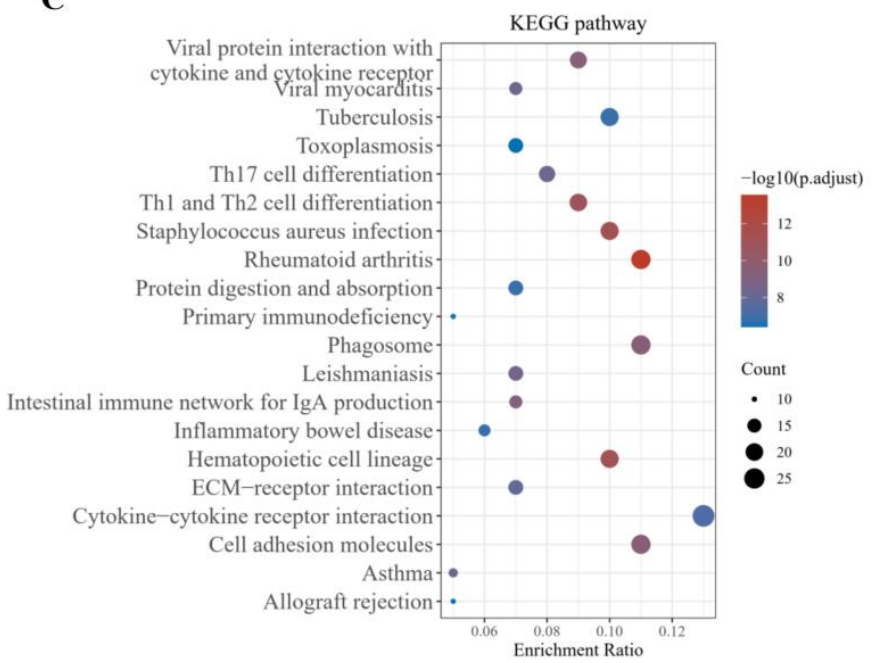

B

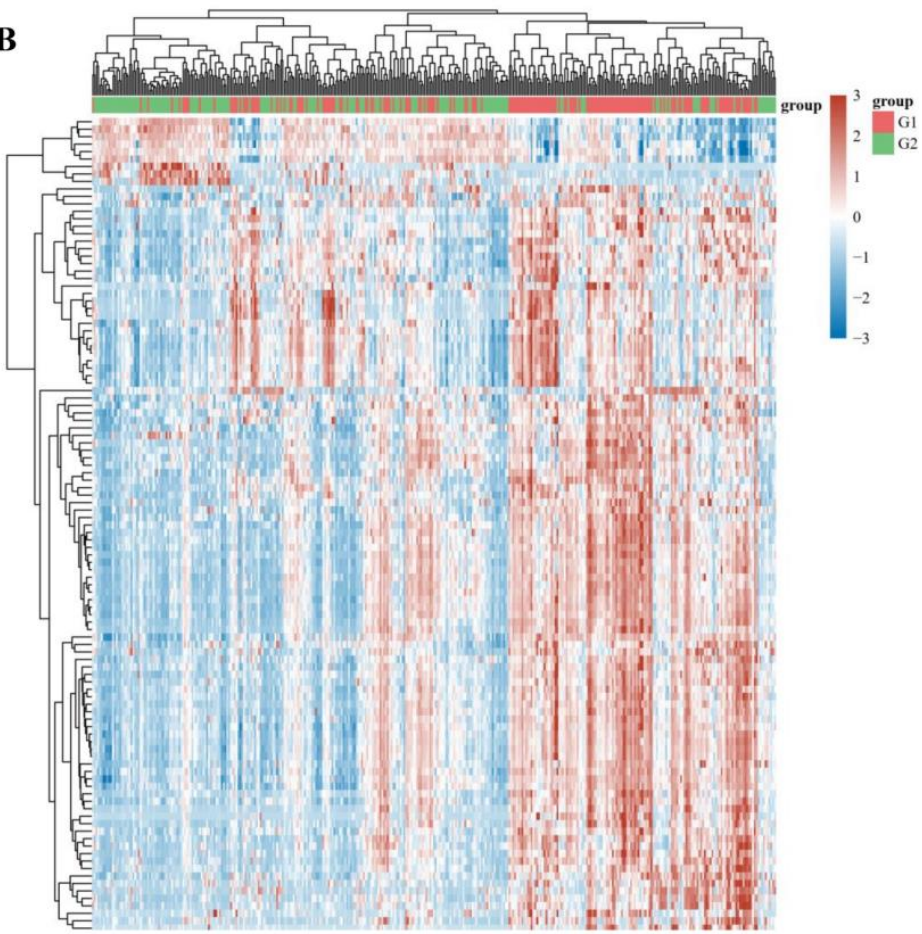

D

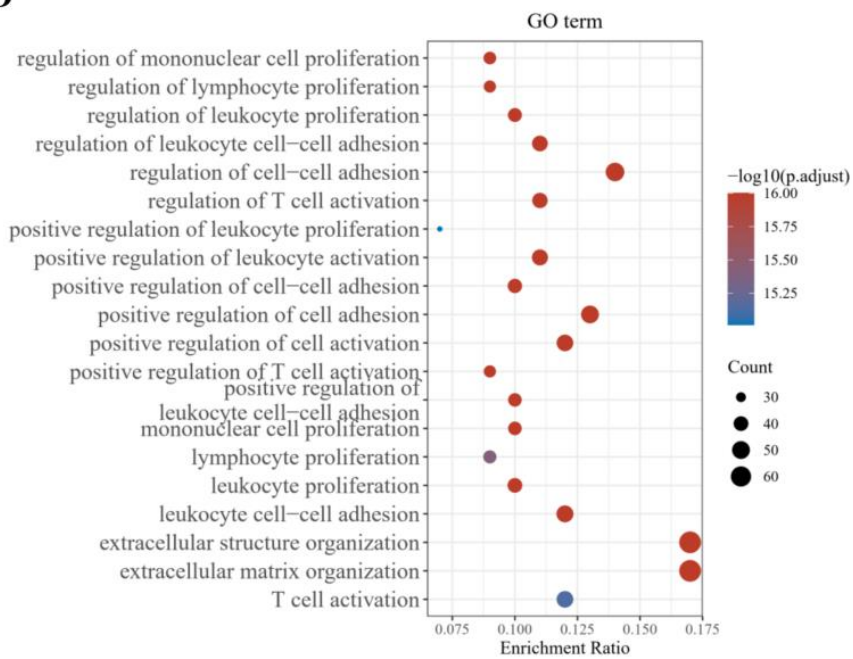

Figure 5. Enrichment analysis for differentially expressed genes. (A) A volcano plot representing the upper quartile (high P2RX1 expression group) and lower quartile (low P2RX1 expression group) of P2RX1 expression. The red points in the plot reflect the high-expressed mRNAs, while the blue points reflect the low-expressed mRNAs with statistical significance. (B) Hierarchical clustering of differentially expressed genes between high P2RX1 expression group and low P2RX1 expression group. (C-D) The KEGG and GO terms enrichment analysis of high P2RX1 expression group. (E-F) The KEGG and GO enrichment analysis of low P2RX1 expression group.

\subsection{P2RX1 is widely associated with a variety of immune infiltrating cells and immune checkpoints in gastrointestinal cancers}

$\mathrm{R}$ packages were used to estimate the data of six kinds of immune cell infiltration in eight types of gastrointestinal tumors from TCGA and GTEx databases. The heatmap showed that there were positive correlations between P2RX1 and different TILs, including B cells, $\mathrm{CD}_{4}{ }^{+} \mathrm{T}$ cells, $\mathrm{CD}_{8}{ }^{+} \mathrm{T}$ cells, neutrophils, macrophages, and dendritic cells (DCs) in COAD, COADREAD, ESCA, LIHC, and STAD (Figure 6A). 
On the whole, there were significant positive associations between P2RX1 and B cells in eight cancer types, $\mathrm{CD}_{4}{ }^{+} \mathrm{T}$ cells in eight cancer types, $\mathrm{CD}_{8}{ }^{+} \mathrm{T}$ cells in six cancer types, neutrophils in seven cancer types, macrophages in six cancer types, and DCs in eight cancer types (Figure 6A). Among all the six TILs, P2RX1 correlated most strongly with DCs in COAD, COADREAD, LIHC, and READ. By analyzing TIMER database, it was found that P2RX1 correlated negatively with tumor purity in the TME of LIHC ( $r$ $=-0.561, P=4.12 \mathrm{e}-30)$. These results are consistent with the above findings, where P2RX1 was found to be positively correlated with all six TILs in LIHC based on TIMER database $(P<0.05)$.

The degree of immune infiltration and the proportion of stromal cells in LIHC were calculated and plotted using the "immunedeconv" package in R software to identify immune infiltration estimations based on TCGA database. The results showed that the expression of P2RX1 was dramatically positively correlated with StromalScore $(r=0.64, P=1.8 \mathrm{e}-43)$ (Figure 6B), ImmuneScore $(r=0.57, P=1.7 \mathrm{e}-32)$ (Figure 6C), and ESTIMATEScore $(r=0.66, P=3.5 \mathrm{e}-46)$ (Figure 6D). These findings indicate that P2RX1 is not only related to immune infiltration but also to the stromal components of TME in LIHC.

Subsequently, correlations between P2RX1 and 47 common immune checkpoint genes adjusted by tumor purity were further analyzed based on TIMER database. Taken as a whole, P2RX1 was found to eb positively correlated with most immune checkpoint genes in gastrointestinal tumors (Figure 6E). In LIHC, the expression of P2RX1 was positively correlated with VEGFB, ARG1, C10orf4, SLAMF7, CTLA4, TIGIT, BTLA, PDCD1 (PD1), HAVCR2, IL10, TGFB1, CD274 (PDL1), IDO1, IFNA2, CD70, ICOSLG, IL1A, TNFSF9, ENTPD1, ITGB2, CCL5, PRF1, CD28, CD40LG, ICOS, CD27, SELP, TNFRSF14, TNFSF4, BTN3A2, TNFRSF9, CD80, CD40, TLR4, ICAM1, GZMA, CXCL9, CXCL10, IFNG, TNFRSF18, IL1B, and TNF $(P<0.05)$ (Figure 6E). Taking into consideration that antibodies linking to PD1, PDL1, and CTLA4 have been identified with therapeutic success in various types of gastrointestinal tumors, P2RX1 was found to be positively correlated with PDCD1 (PD1) and CTLA4 in ESCA, LIHC, READ, COAD, READ, PAAD, and STAD. Positive correlations were also noted between the expression of P2RX1 and CD274 (PDL1) in LIHC, READ, COAD, READ, PAAD, and STAD. To further verify the results, the Gene Expression Profiling Interactive Analysis (GEPIA) database was explored to identify the associations between P2RX1 and several immune checkpoint genes in LIHC. P2RX1 was found to be positively correlated with CD274 $(r=0.35, P=5.3 \mathrm{e}-12)$, PDCD1 $(r=0.52, P=7.3 \mathrm{e}-27)$, and CTLA4 $(r=$ $0.49, P=1.7 \mathrm{e}-23$ ) in LIHC. 

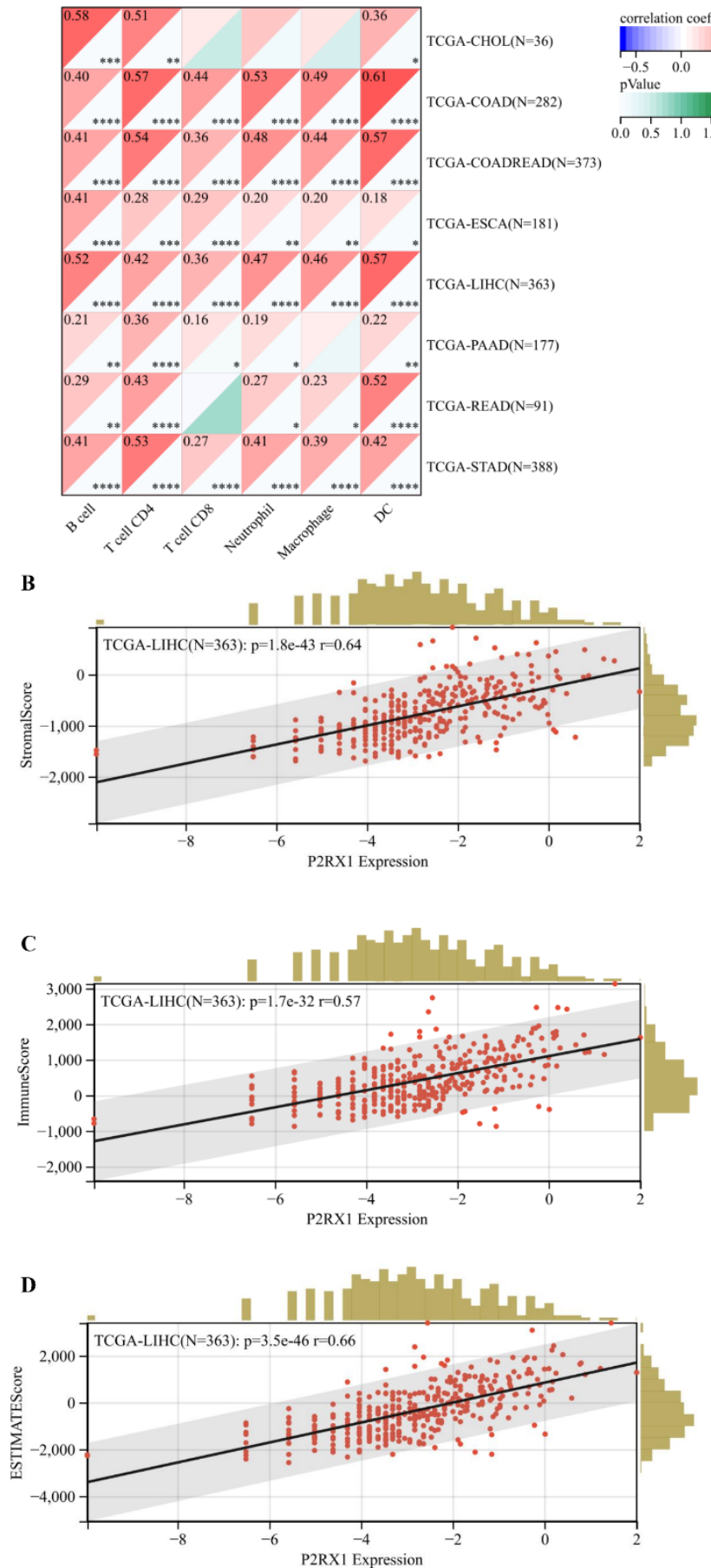
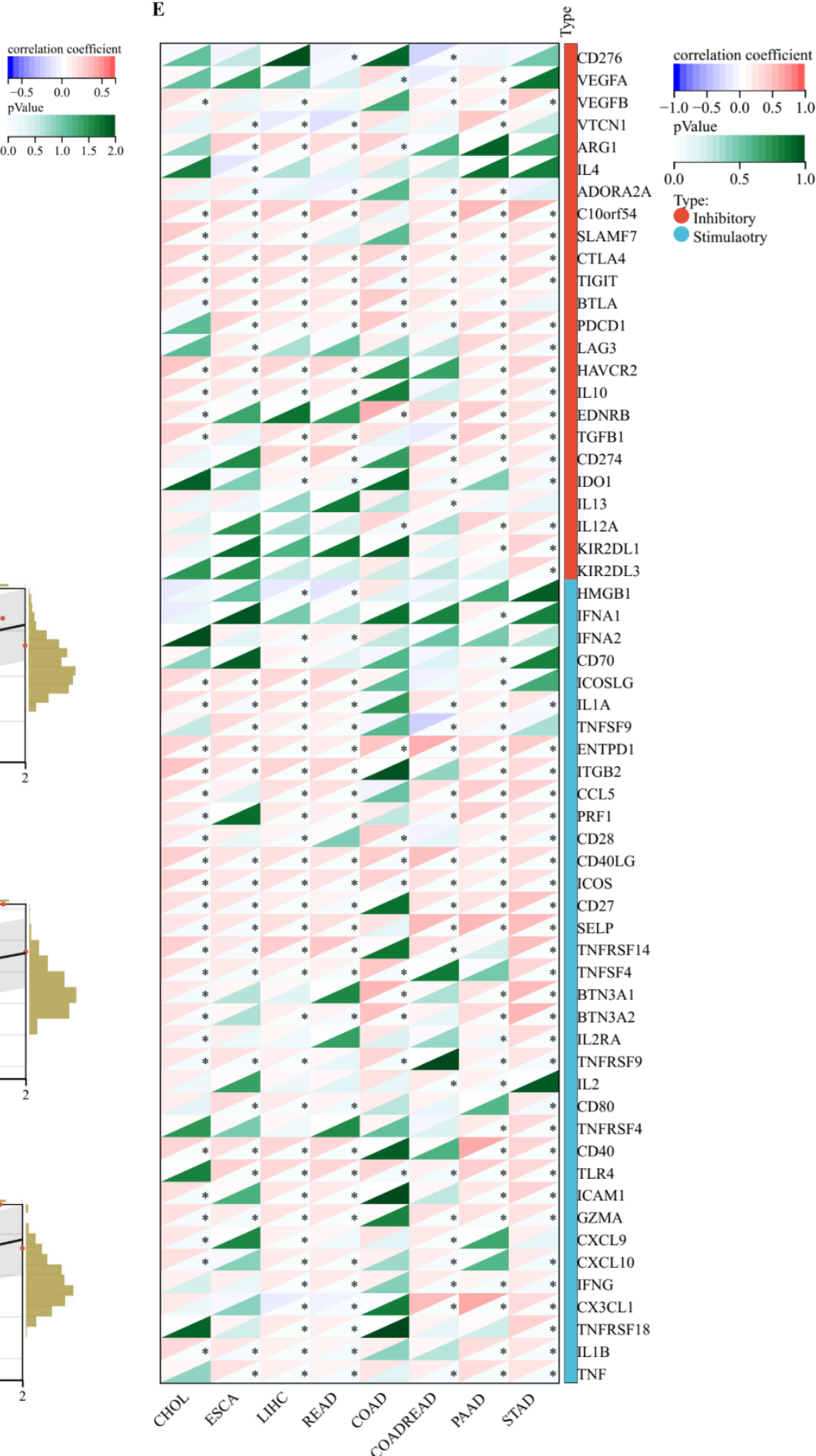

Figure 6. Correlations between P2RX1 expression and TILs in gastrointestinal cancers. (A) A heatmap showing the relationships between P2RX1 and six TILs in eight types of gastrointestinal cancers. (B-D) Associations between P2RX1 and immune infiltration as well as stromal components in patients with LIHC, evaluated by StromalScore, ImmuneScore, and ESTIMATEScore. (E) Correlations between P2RX1 expression and 47 common immune checkpoint genes in eight types of gastrointestinal cancers. Note: TILs, tumor infiltrating lymphocytes; $* P<0.05$.

\section{Discussion}

Purinergic receptor family molecules mainly include 7 members, which are widely involved in many biological processes, such as energy metabolism, inflammatory response, and cell proliferation ${ }^{[14-16]}$. A 
large number of studies have confirmed that ATP-mediated purine signals play an important role in cancer progression and immune regulation ${ }^{[17-20]}$. As a member of P2X receptor family, P2RX1 is involved in mediating synaptic transmission between neurons or smooth muscle, arterial vasoconstriction, reproductive regulation, apoptosis, and so on. However, the crosstalk between P2RX1 expression and gastrointestinal tumors remains unclear. In this study, based on bioinformatics analysis, P2RX1 was found to be significantly downregulated in patients with COAD, ESCA, LIHC, READ, PAAD, and STAD. Combined with the GTEx dataset, the results also showed that P2RX1 decreased significantly in the aforementioned tumors. These results indicate that P2RX1 functions as a tumor suppressor in gastrointestinal cancers. Further investigations based on univariate Cox regression analysis showed that higher expression levels of P2RX1 were associated with better prognosis in LIHC, PAAD, and READ patients. However, P2RX1 was found to be associated with DSS, DFI, and PFI in LIHC patients through Kaplan-Meier analysis. The results also showed that P2RX1 had no associations with the OS of patients with LIHC. Moreover, there were no obvious differences between the expression of P2RX1 and the OS, DSS, DFI, and PFI of patients with PAAD and READ. After analyzing the associations between P2RX1 and various clinicopathological factors of LIHC, the expression of P2RX1 was found to have no correlations with the TNM stage, grade, therapy, gender, and clinical stage of patients with LIHC.

DNA methylation has been reported in the coordination of replication initiation. In this study, P2RX1 was found to be positively correlated with DNMT1, DNMT2A, and DNMT3A in LIHC. This indicates that P2RX1 could promote DNA methylation by upregulating the activity of DNMT enzymes. Further investigations found that cg0101649, cg01783195, cg02021817, cg03943270, cg07396495, and cg13426503 were correlated with patients' overall survival rates in LIHC. Moreover, P2RX1 was noted to be associated with Wilms' tumor 1 associated protein (WTAP) in LIHC. WTAP often acts as an oncogene, and it is associated with the prognosis of patients with LIHC ${ }^{[21]}$. The positive association between the expression of P2RX1 and WTAP in this research promotes the inference of the probability that WTAP induces m6A modification on the mRNA of P2RX1 and coordinates with positive-readers or it links to the 3' UTR or 5' UTR of P2RX1 mRNA, accelerating the stability of P2RX1 mRNA.

Immunotherapy is now being increasingly used in patients with LIHC ${ }^{[22]}$. Combining the enrichment analysis with the immune infiltration results, it was found that P2RX1 extensively participates in the proliferation, differentiation, activation, and regulation of TILs in LIHC. P2RX1 may act as an immune stimulator or promoter in the TME of LIHC. DCs are an attractive target for therapeutic manipulation in LIHC ${ }^{[23]}$. In this study, P2RX1 was found to be dramatically correlated with DCs in many types of gastrointestinal cancers, including COAD, LIHC, and READ. DCs can inhibit T-cell stimulation based on PDL1 signaling pathway ${ }^{[24]}$. The correlation between P2RX1 expression and TILs suggests that P2RX1 regulates gastrointestinal tumor immunity through multiple immune cell populations and regulations. In addition, P2RX1 was also found to be positively correlated with immune/stromal score in LIHC. These results suggest that $\mathrm{P} 2 \mathrm{RX} 1$ has a dual regulatory role on the stromal and immune components in the TME and P2RX1 may participate in the formation and remodeling of the TME. Immune checkpoint inhibitors were identified to be correlated with the treatment outcomes in patients with various cancer types. CTLA4, PD1, and PDL1 can suppress the activities of multiple TILs, including $\mathrm{CD}_{8}{ }^{+} \mathrm{T}$ cells, natural killer cells, and DCs ${ }^{[24]}$. Through the PD1 signaling pathway, tumor cells can escape from the immune surveillance of T cells, and PD1/PDL1 present an important role in tumor immunotherapy ${ }^{[25]}$. In this study, P2RX1 was found to be tightly correlated with PD1, PDL1, and CTLA4 in LIHC, indicating that advanced cancer patients may benefit from immune checkpoint inhibitors with immune-related signature P2RX1.

Nevertheless, although a systematic and complex analysis on P2RX1 was executed and various public datasets as well as R software for cross-verification were explored, some limitations still exist in this study. First, to verify the potential biological role of P2RX1, in vivo/in vitro experiments are essential. Second, 
the screening dataset generated from various databases had differences. These data may have resulted in systematic bias due to the lack of granularity and specificity. Third, direct evidence is required to determine whether P2RX1 can influence the prognosis of different types of gastrointestinal cancers through immune infiltration. Therefore, further experiments are needed to identify the protein expression profiles of P2RX1 in LIHC and biological mechanisms correlated with how P2RX1 regulates progression of tumors and TILs in affecting the prognosis of LIHC patients.

In conclusion, P2RX1 may play a dual role in different types of cancers, including promoting and inhibiting carcinogenesis. In this study, it has been identified and validated that the expression of P2RX1 is downregulated in many gastrointestinal tumors and is significantly linked to the prognosis of patients with LIHC. The high expression of P2RX1 is tightly correlated with the immune infiltration in eight types of gastrointestinal cancers; these TILs include B cells, $\mathrm{CD}_{4}{ }^{+} \mathrm{T}$ cells, $\mathrm{CD}_{8}{ }^{+} \mathrm{T}$ cells, neutrophils, macrophages, and DCs. The bidirectional interaction of P2RX1 in driving or impeding a tumor phenotype is unclear, but it merits exploration.

\section{Acknowledgements}

We would like to thank the previous and present members of Yantai Affiliated Hospital of Binzhou Medical University for analyzing the data and providing constructive feedbacks on the manuscript.

\section{Funding}

This study was supported by the Startup Fund for Scientific Research, Binzhou Medical University (Project Number: BY2019KJ43).

\section{Disclosure statement}

The authors declare that there is no conflict of interest.

\section{Author contributions}

Ting Li and Yunliang Yu designed the research and wrote the manuscript. Ting Li and Pihong Yan analyzed the data. Jiankai Feng and Qiang Li edited the manuscript. Yunliang Yu and Lili Wang revised the figures and tables. All the authors have read and approved the manuscript.

\section{References}

[1] Chen YL, Liu XL, Li L, 2021, Prognostic Value of Low microRNA-34a Expression in Human Gastrointestinal Cancer: A Systematic Review and Meta-analysis. BMC Cancer, 21(1): 63. http://doi:10.1186/s12885-020-07751-y

[2] Dharmawansa KVS, Hoskin DW, Rupasinghe HPV, 2020, Chemopreventive Effect of Dietary Anthocyanins Against Gastrointestinal Cancers: A Review of Recent Advances and Perspectives. Int J Mol Sci, 21(18): 6555. https://doi.org/10.3390/ijms21186555

[3] Hargreave M, Jensen A, Nielsen TSS, et al., 2015, Maternal Use of Fertility Drugs and Risk of Cancer in Children: A Nationwide Population-Based Cohort Study in Denmark. Int J Cancer, 136(8): 19311939.

[4] Yza D, Jiang XB, Ning ZC, et al., 2019, Targeting the Tumour Immune Microenvironment for Cancer Therapy in Human Gastrointestinal Malignancies. Cancer Letters, 458: 123-135. https://doi.org/10.3390/ijms20040840 
[5] Salem ME, Puccini A, Grothey A, et al., 2018, Landscape of Tumor Mutation Load, Mismatch Repair Deficiency, and PD-L1 Expression in a Large Patient Cohort of Gastrointestinal Cancers. Mol Cancer Res, 16(5): 805-812. https://doi.org/10.1158/1541-7786.MCR-17-0735

[6] Najafimehr H, Hajizadeh N, Nazemalhosseini-Mojarad E, et al., 2020, The Role of Human Leukocyte Antigen Class I on Patient Survival in Gastrointestinal Cancers: A Systematic Review and Metaanalysis. Sci Rep, 10(1): 728. https://doi.org/10.1038/s41598-020-57582-X

[7] Ruiz-Bañobre J, Goel A, 2019, DNA Mismatch Repair Deficiency and Immune Checkpoint Inhibitors in Gastrointestinal Cancers. Gastroenterology, 156(4): 890-903. https://doi.org/10.1053/j.gastro.2018.11.071

[8] Zhang WJ, 2021, Effect of P2X Purinergic Receptors in Tumor Progression and as A Potential Target for Anti-tumor Therapy. Purinergic Signal, 17(1): 151-162. https://doi.org/10.1007/s11302-02009761-8

[9] Oury C, Lecut C, Hego A, et al., 2014, Purinergic Control of Inflammation and Thrombosis: Role of P2X1 Receptors. Comput Struct Biotechnol J, 13: 106-110. https://doi.org/10.1016/j.csbj.2014.11.008

[10] Huang Z, Liu P, Zhu L, et al., 2014, P2X1-initiated P38 Signalling Enhances Thromboxane A2-Induced Platelet Secretion and Aggregation. Thromb Haemost, 112(1): 142-50. https://doi: 10.1160/TH13-090726

[11] El Mdawar MB, Maître B, Magnenat S, et al., 2019, The ATP-Gated P2X1 Ion Channel Contributes to the Severity of Antibody-Mediated Transfusion-Related Acute Lung Injury in Mice. Sci Rep, 9(1): 5159. https://doi.org/10.1038/s41598-019-41742-9

[12] Yang X, Lu B, Sun X, et al., 2018, ANP32A Regulates Histone H3 Acetylation and Promotes Leukemogenesis. Leukemia, 32(7): 1587-1597. https://doi: 10.1038/s41375-018-0010-7

[13] Ma C, Luo H, Cao J, et al., 2020, Identification of a Novel Tumor Microenvironment-Associated EightGene Signature for Prognosis Prediction in Lung Adenocarcinoma. Front Mol Biosci, 7: 571641. https://doi.org/10.3389/fmolb.2020.571641

[14] Wang X, Hu LP, Qin WT, et al., 2021, Identification of A Subset of Immunosuppressive P2RX1Negative Neutrophils in Pancreatic Cancer Liver Metastasis. Nat Commun, 12(1): 174. https://doi.org/10.1038/s41467-020-20447-y

[15] Wang X, Liu D, Qin W, et al., 2021, P2RX1-Involved Glycolytic Metabolism Supports Neutrophil Activation in Acute Pancreatitis. Front Immunol, 11: 549179. https://doi.org/10.3389/fimmu.2020.549179

[16] Wang X, Yuan X, Su Y, et al., 2021, Targeting Purinergic Receptor P2RX1 Modulates Intestinal Microbiota and Alleviates Inflammation in Colitis. Front Immunol, 12: 696766. https://doi.org/10.3389/fimmu.2021.696766

[17] Alharbi AF, Parrington J, 2021, The Role of Genetic Polymorphisms in Endolysosomal Ion Channels TPC2 and P2RX4 in Cancer Pathogenesis, Prognosis, and Diagnosis: A Genetic Association in the UK Biobank. NPJ Genom Med, 6(1): 58. https://doi.org/10.1038/s41525-021-00221-9

[18] Romagnani A, Rottoli E, Mazza EMC, et al., 2020, P2X7 Receptor Activity Limits Accumulation of T Cells within Tumors. Cancer Res, 80(18): 3906-3919. https://doi.org/10.1158/0008-5472.CAN-193807

[19] Jain S, Jacobson KA, 2021, Purinergic Signaling in Liver Pathophysiology. Front Endocrinol (Lausanne), 12: 718429. https://doi.org/10.3389/fendo.2021.718429

[20] Scarpellino G, Genova T, Avanzato D, et al., 2019, Purinergic Calcium Signals in Tumor-Derived 
Endothelium. Cancers (Basel), 11(6): 766. https://doi.org/10.3390/cancers11060766

[21] Liu W, Gao X, Chen X, et al., 2021, miR-139-5p Loss-Mediated WTAP Activation Contributes to Hepatocellular Carcinoma Progression by Promoting the Epithelial to Mesenchymal Transition. Front Oncol, 11: 611544. https://doi.org/10.3389/fonc.2021.611544

[22] Li L, Song X, Lv Y, et al., 2020, Landscape of Associations Between Long Non-coding RNAs and Infiltrating Immune Cells in Liver Hepatocellular Carcinoma. J Cell Mol Med, 24(19): 11243-11253. https://doi.org/10.1111/jcmm.15690

[23] Hsiao YW, Chiu LT, Chen CH, et al., 2019, Tumor-Infiltrating Leukocyte Composition and Prognostic Power in Hepatitis B- and Hepatitis C-Related Hepatocellular Carcinomas. Genes (Basel), 10(8): 630. https://doi.org/10.3390/genes 10080630

[24] Bolis M, Paroni G, Fratelli M, et al., 2020, All-Trans Retinoic Acid Stimulates Viral Mimicry, Interferon Responses and Antigen Presentation in Breast-Cancer Cells. Cancers (Basel), 12(5): 1169. https://doi.org/10.3390/cancers12051169

[25] Li Z, Suo B, Long G, et al., 2020, Exosomal miRNA-16-5p Derived from M1 Macrophages Enhances T Cell-Dependent Immune Response by Regulating PD-L1 in Gastric Cancer. Front Cell Dev Biol, 8: 572689. https://doi.org/10.3389/fcell.2020.572689 